\title{
Probing the cosmic acceleration history and the properties of dark energy from the ESSENCE supernova data with a model independent method
}

\author{
Puxun $\mathrm{Wu}^{1,2}$ and Hongwei $\mathrm{Yu}^{3}$ \\ ${ }^{1}$ Department of Physics and Tsinghua Center for Astrophysics, \\ Tsinghua University, Beijing 100084, China \\ ${ }^{2}$ Institute of Math-Physics and School of Sciences, \\ Central South University of Forestry and Technology, Changsha, Hunan 410004, China \\ ${ }^{3}$ Department of Physics and Institute of Physics, \\ Hunan Normal University, Changsha, Hunan 410081, China
}

\begin{abstract}
With a model independent method the expansion history $H(z)$, the deceleration parameter $q(z)$ of the universe and the equation of state $w(z)$ for the dark energy are reconstructed directly from the 192 Sne Ia data points, which contain the new ESSENCE Sne Ia data and the high redshift Sne Ia data. We find that the evolving properties of $q(z)$ and $w(z)$ reconstructed from the 192 Sne Ia data seem to be weaker than that obtained from the Gold set, but stronger than that from the SNLS set. With a combination of the 192 Sne Ia and BAO data, a tight constraint on $\Omega_{m 0}$ is obtained. At the $1 \sigma$ confidence level $\Omega_{m 0}=0.278_{-0.023}^{+0.024}$, which is highly consistent with that from the Gold+BAO and SNLS+BAO.

PACS numbers: $98.80 . \mathrm{Es}, 98.80 .-\mathrm{k}$
\end{abstract}




\section{INTRODUCTION}

In order to explain the present cosmic accelerating expansion discovered firstly from the Type Ia Supernovae (Sne Ia) [1, 2, 3, 4, 5, 6], dark energy (see [7, 8, 9, 10] for recent reviews) is usually assumed to exist in the universe. Dark energy is an exotic energy component with negative pressure, and presumably began to dominate the evolution of the universe only recently. Although it has been studied for nearly one decade, its nature is still puzzling.

In general there are two kinds of methods to reconstruct the properties of dark energy from the observation data directly. One is to assume an arbitrary parametrization for the equation of state of dark energy, $w(z)$, the potential of dark energy, $V(z)$, the Hubble parameter, $H(z)$, or the luminosity distance $d_{L}(z)$ with some arbitrary constants. By determining these constants from the observational data, we can obtain the evolving properties of dark energy. Different ways of parametrization have been discussed in Refs. [11, 12, 13]. The other is the no-parametric method, which usually involves directly smoothing either $d_{L}$, or some other quantity with some characteristic smoothing scale. Currently there are many different models of implementing this approach [14, 15, 16]

Recently, based on smoothing the noise of supernova data over redshift, the authors in Refs. [15, 16] suggested a no-parametric method in a model independent manner to reconstruct the expansion history of our universe and the evolving properties of dark energy. In Ref. [16] two kinds of supernova data: 182 Gold dataset and 115 SNLS dataset are used firstly to reconstruct the Hubble parameter $h(z)\left(h(z)=H(z) / H_{0}\right)$ and the deceleration parameter $q(z)$. It was found that both data sets give $q(0)<0$, which means the universe is undergoing an accelerating expansion, while the Gold set seems to favor a later entering of this accelerating era than the SNLS one. However Gold and SNLS give a good consistent constraint on the present matter density parameter $\Omega_{m 0}\left(\Omega_{m 0} \approx 0.276 \pm 0.023\right)$ when combined with the baryonic acoustic oscillation peak obtained from the large scale correlation function of luminous red galaxy in the Sloan Digital Sky Survey (SDSS) [17]. The w(z) was also discussed with both kinds of SNe Ia datasets, and it was found, in agreement with that

obtained in Refs. [18, 19, 20] using some parameterized models, the Gold slightly favors an dynamically evolving dark energy with a crossing of phantom divide line while the SNLS does not. However, in this method, the present value of Hubble parameter, $H_{0}$, is needed prior or should be marginalized over. Since the value of $H_{0}$ from different observation data 
seems to be inconsistent and doing the marginalization wastes the compute resources, in this paper, we firstly generalize this model independent approach to eliminate the impact of $H_{0}$ and then reconstruct the cosmic expansion history, $H(z)$, the deceleration parameter, $q(z)$, and the equation of state for dark energy $w(z)$ from the new ESSENCE Sne Ia data. Beside the 162 data points given in table 9 in Ref. [6], which contains 60 ESSENCE Sne Ia, 57 SNLS Sne Ia and 45 nearby Sne Ia, we add 30 Sne Ia detected at $0.216<z<1.755$ by the Hubble Space Telescope [4].

\section{THE METHOD}

Following a well known procedure in the analysis of large scale structure, Shafieloo et al. [15, 16] use a Gaussian smoothing function rather than the top hat smoothing function to smooth the noise of the Sne Ia data directly. In order to obtain the important information of interested cosmological parameters expediently, $\ln d_{L}(z)$ rather than the luminosity distance $d_{L}(z)$ or distance module $\mu(z)$ is studied by the following iterative method

$$
\ln d_{L}(z)_{n}^{s}=\ln d_{L}(z)_{n-1}^{s}+N(z) \sum_{i}\left(\ln d_{L}^{o b s}\left(z_{i}\right)-\ln d_{L}\left(z_{i}\right)_{n-1}^{s}\right) \exp \left[-\frac{\ln ^{2}\left(\frac{1+z}{1+z_{i}}\right)}{2 \triangle^{2}}\right]
$$

with a normalization parameter

$$
N(z)^{-1}=\sum_{i} \exp \left[-\frac{\ln ^{2}\left(\frac{1+z}{1+z_{i}}\right)}{2 \triangle^{2}}\right]
$$

In Eqs. $(1,2) \triangle$ is a quantity needed to be given prior. Since a large value of $\triangle$ leads to a smooth result but depresses the accuracy of reconstruction, and inversely for a small value of $\triangle$. So it is important to choose a reasonable value of $\triangle$. Here, as in Ref. [16], we choose $\triangle=0.6$. In Eq.(1), $d_{L}(z)_{n}^{s}$ represents the smoothed luminosity distance at any redshift $z$ after $n$ iteration. When $n=1 d_{L}(z)_{0}^{s}$ denotes a guess background model and it has been shown that the results are not sensitive to the chosen vaule of $\Delta$ and the assumed initial guess model [16]. In this paper we use a $w C D M$ model with $w=-0.9$ and $\Omega_{m 0}=0.28$ as this guess background model. $\ln d_{L}^{o b s}\left(z_{i}\right)$ is the observed one from the Sne Ia and can be expressed as:

$$
\ln d_{L}^{o b s}\left(z_{i}\right)=\frac{\ln 10}{5}\left[\mu^{o b s}\left(z_{i}\right)-42.38\right]+\ln h \equiv \ln f^{o b s}\left(z_{i}\right)+\ln h
$$


Here $h=H_{0} / 100$ and $\mu^{o b s}$ is the observed distance module of Sne Ia. Apparently using the above method the nuisance parameter $h$ needs to be given prior or marginalized over. Now we generalize this method to eliminate the impact of $h$. Substituting Eq. (3) into Eq. (1), we obtain that

$$
\ln d_{L}(z)_{n}^{s}=\ln d_{L}(z)_{n-1}^{s}+N(z) \sum_{i}\left(\ln f^{o b s}\left(z_{i}\right)-\ln d_{L}\left(z_{i}\right)_{n-1}^{s}\right) \exp \left[-\frac{\ln ^{2}\left(\frac{1+z}{1+z_{i}}\right)}{2 \triangle^{2}}\right]+\ln h \text {. }
$$

If defining $\ln d_{L}(z)_{n}^{s}=\ln f(z)_{n}^{s}+\ln h$, it is easy to see

$$
\ln f(z)_{n}^{s}=\ln f(z)_{n-1}^{s}+N(z) \sum_{i}\left(\ln f^{o b s}\left(z_{i}\right)-\ln f(z)_{n-1}^{s}\right) \exp \left[-\frac{\ln ^{2}\left(\frac{1+z}{1+z_{i}}\right)}{2 \triangle^{2}}\right] .
$$

When $n=1$

$$
\begin{aligned}
\ln f(z)_{1}^{s} & =\ln f(z)_{0}^{s}+N(z) \sum_{i}\left(\ln f^{o b s}\left(z_{i}\right)-\ln f(z)_{0}^{s}\right) \exp \left[-\frac{\ln ^{2}\left(\frac{1+z}{1+z_{i}}\right)}{2 \triangle^{2}}\right] \\
& =\ln d_{L}(z)_{0}^{s}+N(z) \sum_{i}\left(\ln f^{o b s}\left(z_{i}\right)-\ln d_{L}(z)_{0}^{s}\right) \exp \left[-\frac{\ln ^{2}\left(\frac{1+z}{1+z_{i}}\right)}{2 \triangle^{2}}\right] .
\end{aligned}
$$

Here $d_{L}(z)_{0}^{s}$ is the luminosity distance of the suggested background model. Different from Ref.[16] to iterate through Eq.(1), we use Eq. (5) to obtain the smoothed results. The advantage of doing so is that result is independent of $h$ (or $H_{0}$ ). In order to determine whether we obtain a best fit model after some iteration, we calculate, after each iteration, $\chi^{2}$ :

$$
\chi_{n}^{2}=\sum_{i} \frac{\left(\mu\left(z_{i}\right)_{n}-\mu^{o b s}\left(z_{i}\right)\right)^{2}}{\sigma_{\mu_{o b s, i}}^{2}}
$$

Once this $\chi_{n}^{2}$ reaches its minimum value we stop the iterative process and get the best fit result.

By differentiating the smoothed luminosity distance we can find the Hubble parameter, $H(z),(\operatorname{not} h(z))$

$$
H(z)=\left[\frac{d}{d z}\left(\frac{100 f(z)}{1+z}\right)\right]^{-1}
$$

which contains the information of $H_{0}$. Then the deceleration parameter $q(z)$ of the universe and the equation of state $w(z)$ of dark energy can be obtained:

$$
\begin{gathered}
q(z)=(1+z) \frac{H^{\prime}(z)}{H(z)}-1, \\
w(z)=\frac{[2(1+z) / 3] H^{\prime} / H-1}{1-\left(H_{0} / H\right)^{2} \Omega_{m 0}(1+z)^{2}} .
\end{gathered}
$$




\section{THE RESULTS}

The ESSENCE program (Equation of State: Supernovae trace Cosmic Expansionan NOAO Survey Program) is designed to measure the history of cosmic expansion over the past 5 billion years. The four year data was released in Ref. [6], which contains 60 Sne Ia points. Here we use the 162 data points given in table 9 in Ref. [6], which contains 60 ESSENCE Sne Ia, 57 SNLS Sne Ia and 45 nearby Sne Ia. In addition, as in Ref. [21], we add 30 Sne Ia detected at $0.216<z<1.755$ by the Hubble Space Telescope [4].

Using these 192 Sne Ia data points, we find when $n=42$ a minimum value of $\chi^{2}$ is obtained which can be seen from the Fig. (1). In Fig. (2) we show the reconstructed result of the Hubble parameter $H(z)$ with the likelihood within $1 \sigma$. The red line is the best fit result and when $z=0$ the best fit value of $H_{0}$ is $H_{0}=65.5$. Fig. (3) shows the evolving curves of reconstructed $q(z)$ with $1 \sigma$ error bar. It is easy to see that the universe is undergoing an accelerating expansion since the present value of $q(z)$ is less than zero, and the phase transition from deceleration to acceleration occurs at redshift $z \sim 0.55-0.73$ within $1 \sigma$, which is slightly later than that obtained from Gold set $(z \sim 0.38-0.48)$ but earlier than that from SNLS set $(z>0.7)[16]$.

Fig. (4) shows the constraint on the present matter density parameter $\Omega_{m 0}$ with $H_{0}=65.5$ by combining the ESSENCE Sne Ia and baryonic acoustic oscillation(BAO) peak obtained from the large scale correlation function of luminous red galaxy in the Sloan Digital Sky Survey (SDSS). For BAO data we use a model-independent dimensionless parameter $A$ defined as

$$
A=\frac{\sqrt{\Omega_{m 0}}}{h\left(z_{1}\right)^{1 / 3}}\left[\frac{1}{z_{1}} \int_{0}^{z_{1}} \frac{d z}{h(z)}\right]^{2 / 3},
$$

for a flat universe, where $z_{1}=0.35$ and $A$ is measured to be $A=0.469\left(\frac{n}{0.96}\right)^{-0.35} \pm 0.017$ [17]. Here $n$ is the spectral index of the primordial power spectrum and the WMAP3 gives $n=0.951$ [22]. Clearly the ESSENCE Sne Ia and BAO give a strong constraint on $\Omega_{m 0}$. At the $1 \sigma$ confidence level we obtain $\Omega_{m 0}=0.278_{-0.023}^{+0.024}$, which is highly consistent with that obtained from Gold $+\mathrm{BAO}$ and SNLS+BAO $\left(\Omega_{m 0}=0.276 \pm 0.023\right)$ [16].

In Fig. (5) we plot the evolving behavior of $w(z)$ with a marginalization of $\Omega_{m 0}$ over $\Omega_{m 0}=0.278_{-0.023}^{+0.024}$. The best fit (red) line shows that the ESSENCE data slightly favors an evolving dark energy with a crossing of phantom divide line at the near past, however 
this evolving property is weaker than that obtained from Gold data but stronger than that from SNSL set obtained in Ref. [16]. In addition, from Figs. (3) and (5) we find that the stringent constraint on $w(z)$ and $q(z)$ happens around redshift $z \sim 0.5$, which is consistent with that obtained in Refs. [20, 23] with some parameterized models.

\section{CONCLUSION}

In this paper, with a model independent method we have reconstructed the cosmic expansion history and the properties of dark energy from recent ESSENCE Sne Ia data. We firstly obtain the evolution of $H(z)$. Then the cosmic deceleration parameter $q(z)$ and the equation of state $w(z)$ of dark energy are reconstructed. Our results show that their evolutionary property reconstructed from ESSENCE data is weaker than that from Gold set, but is stronger than that from the SNSL one. Combining the ESSENCE Sne Ia and the BAO data, a tight constraint on $\Omega_{m 0}$ is obtained. At a $1 \sigma$ confidence level $\Omega_{m 0}=0.278_{-0.023}^{+0.024}$, which is highly consistent with that obtained from Gold+BAO and SNLS+BAO. Remarkably, as that obtained with some parameterized model [20, 23], the tight constraints on $w(z)$ and $q(z)$ seem to happen at about $z \sim 0.5$.

\section{Acknowledgments}

This work was supported in part by the National Natural Science Foundation of China under Grants No. 10575035, 10775050 and 10705055, the Program for NCET under Grant No. 04-0784, the SRFDP under Grant No. 20070542002, the Youth Scientific Research Fund

of Hunan Provincial Education Department under Grant No. 07B085, and the Foundation of CSUFT under Grant No. 06Y020.

[1] S. Perlmutter, et al., 1999 Astrophys. J. 517565

[2] A. G. Riess, et al., 1998 Astron. J. 1161009

[3] A. G. Riess, et al., 2004 Astrophys. J. 607665

[4] A. G. Riess, et al., 2007 Astrophys. J. 65998

[5] P. Astier et al., 2006 Astron. Astrophys. 44731 
[6] W. M. Wood-Vasey et al., 2007 arXiv: astro-ph/0701041

[7] T. Padmanabhan, 2006 AIP Conf. Proc. 861179

[8] E. J. Copeland, M. Sami and S. Tsujikawa, 2006 Int. J. Mod. Phys. D 151753

[9] V. Sahni and A. Starobinsky, 2006 Int. J. Mod. Phys. D 152105

[10] L. Perivolaropoulos, 2006 arXiv: astro-ph/0601014

[11] A. A. Starobinsky, 1998 JETP Lett. 68757

D. Huterer and M. S. Turner, 1999 Phys. Rev. D 60081301

P. Astier, 2000 arXiv:astro-ph/0008306

T. Chiba and T. Nakamura, 2000 Phys. Rev. D 62121301

J. Weller and A. Albrecht, 2002 Phys. Rev. D 65103512

I. Maor, R. Brustein, J. McMahon and P. J. Steinhardt, 2002 Phys. Rev. D 65123003

M. Chevallier, D. Polarski, 2001 Int. J. Mod. Phy. D. 10213

E. V. Linder, 2003 Phys. Rev. Lett. 90091301

H. K. Jassal, J .S. Bagla and T. Padmanabhan, 2005 Mon. Not. Roy. Astron. Soc. 356 L11

[12] V. Sahni, T. D. Saini, A. A. Starobinsky and U. Alam, 2003 JETP lett. 77201

U. Alam, V. Sahni, T. D. Saini and A. A. Starobinsky, 2003 Mon. not. Roy. Ast. Soc. 344 1057

U. Alam, V. Sahni, T. D. Saini and A. A. Starobinsky, 2004 astro-ph/0406672 T. D. Saini, S. Raychaudhury, V. Sahni and A. A. Starobinsky, 2000 Phys. Rev. Lett. 851162

T. D. Saini, J. Weller and S. L. Bridle, 2004 Mon. Not. Roy. Ast. Soc. 348603

U. Alam, V. Sahni and A. A. Starobinsky, 2004 J. Cosmol. Astropart.Phys. 0406008

U. Alam, V. Sahni, T. D. Saini and A. A. Starobinsky, 2004 Mon. Not. Roy. Ast. Soc. 354 275

[13] B. Gerke and G. Efstathiou, 2002 Mon. Not. Roy. Ast. Soc. 33533

I. Maor, R. Brustein, J. McMahon and P. J. Steinhardt, 2002 Phys. Rev. D 65123003

P. S. Corasaniti and E. J. Copeland, 2003 Phys. Rev. D 67063521

Y. Wang and P. Mukherjee, 2004 Astroph. J. 606654

S. Nesseris and L. Perivolaroupolos, 2004 Phys. Rev. D 70043531

T. Roy Choudhury and T. Padmanabhan, 2005 Astron. Astrophys. 429807

Y. Gong, 2005 Int. J. Mod. Phys. D 14599

C. Wetterich, 2004 Phys. Lett. B 59417 
P. Wu and H. Yu, 2006 Phys. Lett. B 643315

Z. K. Guo, N. Ohta and Y. Z. Zhang, 2005 Phys. Rev. D 72023504

Z. K. Guo, N. Ohta and Y. Z. Zhang, 2007 Mod. Phys. Lett. A 22883

J. Simon, L. Verde and R. Jimenez, 2005 Phys. Rev. D 71123001

[14] Y. Wang and G. Lovelace, 2001 Astroph. J. 562 L115

T. D. Saini, 2003 Mon. Not. Roy. Ast. Soc. 344129

A. Daly and S. G. Djorgovsky, 2003 Astroph. J. 5979

R. A. Daly and S. G. Djorgovsky, 2004 Astroph. J. 612652

R. A. Daly and S. G. Djorgovsky, 2006 astro-ph/0609791

Y. Wang and M. Tegmark, 2004 Phys. Rev. Lett. 92241302

Y. Wang and M. Tegmark, 2005 Phys. Rev. D 71103513

R. A. Daly and S. G. Djorgovsky, 2005 astro-ph/0512576

D. Huterer and A. Cooray, 2005, Phys. Rev. D 71023506

D. Huterer and G. Starkman, 2003, Phys. Rev. Lett. 90031301

S. Fay and R. Tavakol, 2006 Phys. Rev. D 74083513

[15] A. Shafieloo, U. Alam, V. Sahni and A. Starobinsky, 2006 Mon. Not. R. Astron. Soc. 366 1081

[16] A. Shafieloo, 2007 Mon. Not. R. Astron. Soc. 3801573

[17] D. J. Eisenstein, et al., 2005 Astrophys. J. 633560

[18] S. Nesseris and L. Perivolaropoulos, 2007 J. Cosmol. Astropart. Phys. 01018

[19] U. Alam, V. Sahni and A. Starobinsky, 2007 J. Cosmol. Astropart. Phys. 02011

[20] P. Wu and H. Yu, 2007 J. Cosmol. Astropart. Phys. 10014

[21] T. M. Davis, et al., arXiv:astro-ph/0701510

Barger V, Gao Y and Marfatia D, 2007 Phys.Lett. B 648127

[22] D. N. Spergel, et al., 2007 Astrophys. J. Supp. 170377

[23] Y. G. Gong, 2005 Class. Quantum Grav. 222121

Y. G. Gong and Y.Z. Zhang, 2005 Phys. Rev. D 72043518

M. Chevallier and D. Polarski, 2001 Int. J. Mod. Phys. D 10213

Y. Gong and A. Wang, 2007 Phys. Rev. D 75043520 


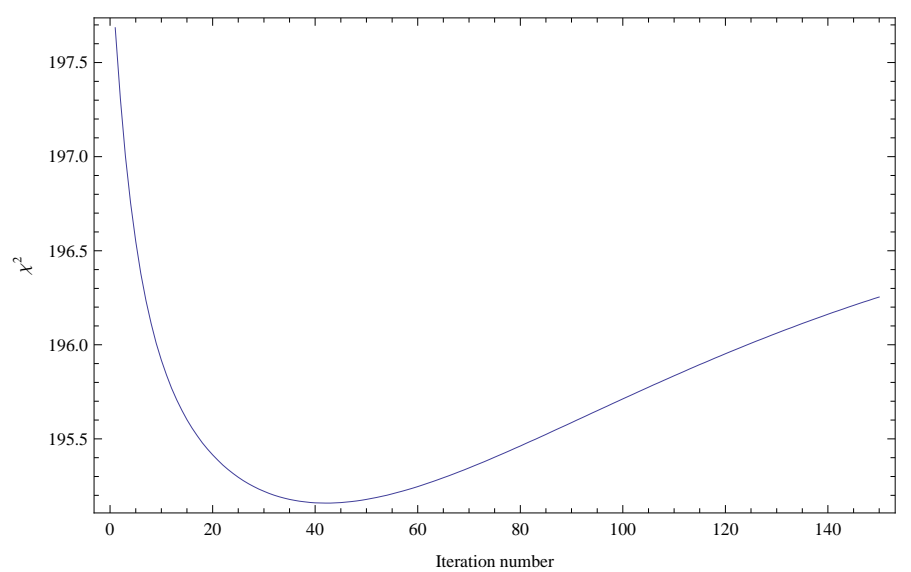

FIG. 1: Computed $\chi^{2}$ for the reconstructed results at each iteration for the 192 Sne Ia data.



FIG. 2: The reconstructed evolutionary curves of the Hubble parameter $H(z)$ with the likelihood within $1 \sigma$. The red line is the best recovered result. 


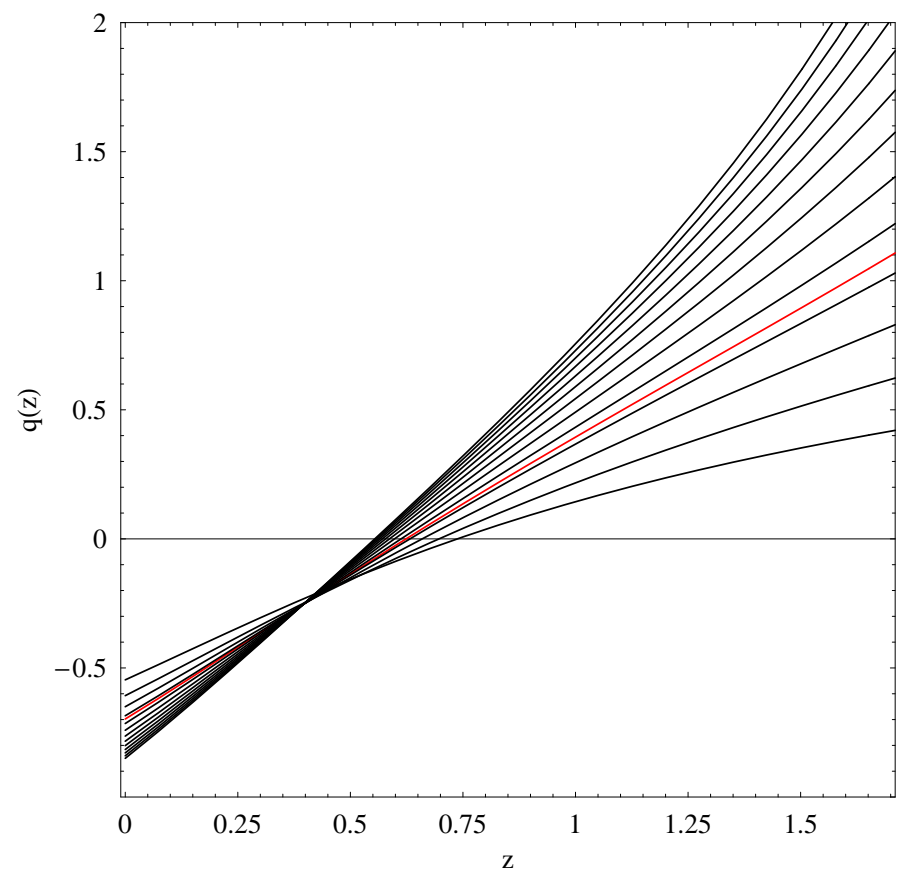

FIG. 3: The reconstructed evolutionary curves of the deceleration parameter $q(z)$ with the likelihood within $1 \sigma$. The red line is the best recovered result.

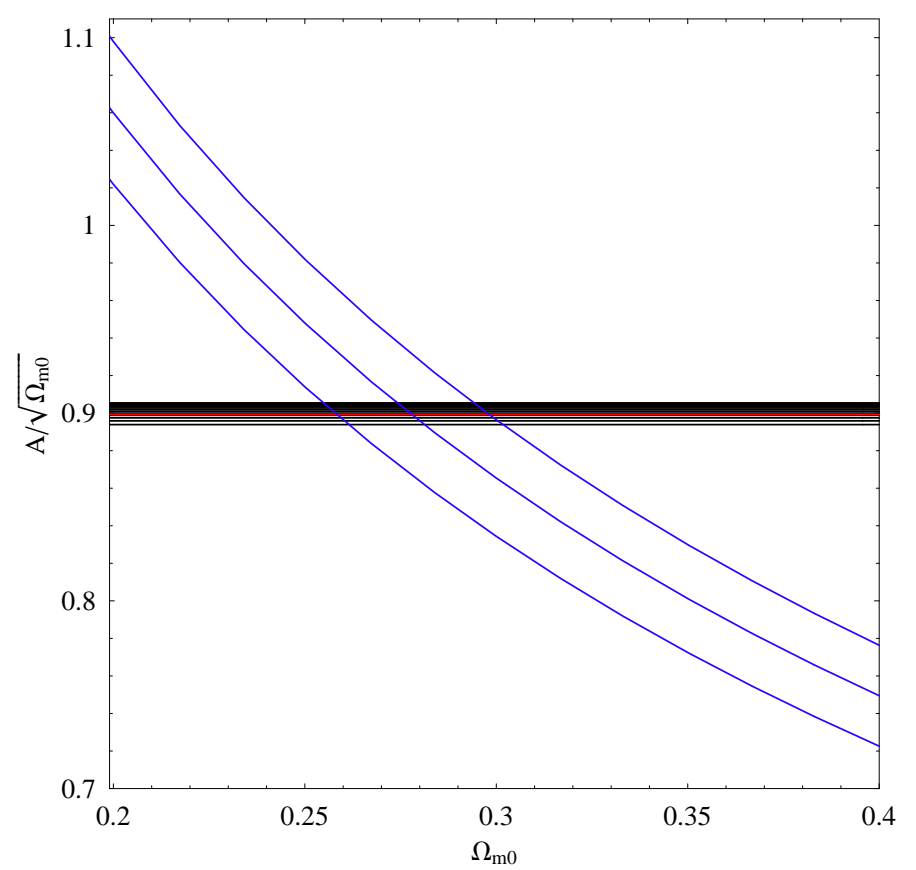

FIG. 4: The constraint on $\Omega_{m 0}$ from the combination of 192 Sne Ia and BAO data. The red and black lines show the derived value of $A / \sqrt{\Omega_{m 0}}$ from the 192 Sne Ia dataset within $1 \sigma$ and the blue lines are the results from the BAO data. 


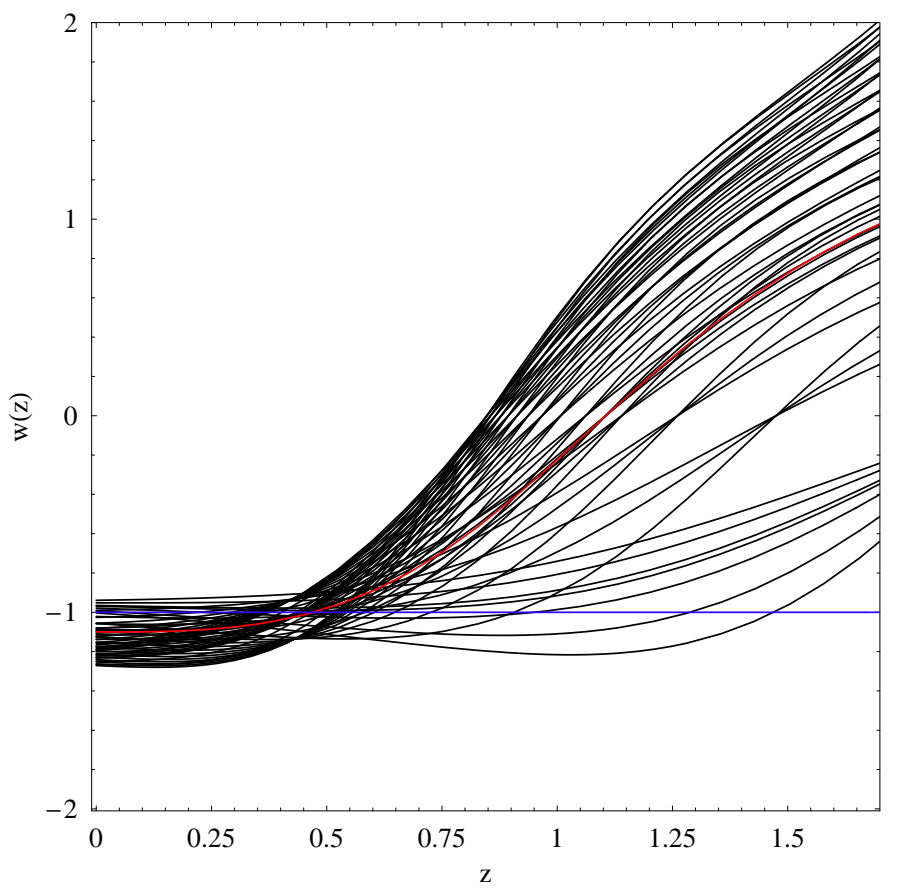

FIG. 5: The reconstructed evolutionary curves of the equation of state of dark energy, $w(z)$, within $1 \sigma$ with a marginalization over $\Omega_{m 0}=0.278_{-0.023}^{+0.024}$. 